\title{
Análisis de la relación circular entre reputación corporativa y creación de valor económico patrimonial
}

\section{pp. 85-95}

\author{
JULIO DAZA-IZQUIERDOa
}

\begin{abstract}
RESUMEN Determinar si las compañías deben incorporar aspectos sociales en sus estrategias de gestión conducentes a satisfacer las expectativas de sus distintos stakeholders presenta posturas teóricas y evidencia empírica previa tanto a favor como en contra. El objetivo del presente estudio consiste en analizar la relación existente entre reputación corporativa y creación de valor económico patrimonial en las empresas cotizadas en el mercado bursátil español durante el periodo 2000-2012 aplicando una metodología de datos de panel. Los resultados obtenidos nos indican que la reputación influye positivamente en la creación de valor económico patrimonial, y que, al mismo tiempo, un mayor valor empresarial repercute en una mayor reputación corporativa, dando muestras de una relación bidireccional que retroalimenta las dos variables objeto de estudio. Estos resultados tienen importantes implicaciones para la gestión, ya que legitiman la integración de prácticas de responsabilidad social corporativa en las grandes empresas españolas cotizadas en bolsa.
\end{abstract}

PALABRAS CLAVE accionistas, creación de valor, datos de panel, grupos de interés, ratio q de Tobin, reputación corporativa.

\section{HISTORIA DEL ARTÍCULO}

¿CÓMO CITAR?:

Daza-Izquierdo, J. (2015). Análisis de la relación circular entre reputación corporativa y creación de valor económico patrimonial. Perspectiva Empresarial, 2(1), 85-95. http:// dx.doi.org/10.16967\%2Frpe. v2n1a6

RECIBIDO: 6 de noviembre de 2014 APROBADO: 16 de enero de 2015 CORRESPONDENCIA: Julio Daza-Izquierdo. Av. Elvas s/n 06071 Badajoz, España.

a Departamento de Economía Financiera y Contabilidad, Universidad de Extremadura, España. Correo electrónico: juliodaza@unex.es 


\section{Analysis of the circular relationship between corporate reputation and the creation of patrimonial economic value}

ABSTRACT Determining if companies should incorporate social aspects in their management strategies to satisfy the expectations of their different stakeholders presents theoretical postures and previous empirical evidence both for and against it. The objective of this study consists of analyzing the existing relationship between corporate reputation and the creation of patrimonial economic value in the companies sought-after in the Spanish stock market in the 2000-2012 period by applying a methodology of panel data. The obtained results show us that reputation has a positive influence in the creation of patrimonial economic value and that, at the same time, a higher company value leads to a higher corporate reputation showing a bi-directional relationship, which provides feedback to the two variables that are the subject of this study. These results have important implications for

¿CÓMO CITO EL ARTÍCULO? HOW TO CITE THIS PAPER?

\section{CHICAGO:}

Daza-Izquierdo, Julio. "Análisis de la relación circular entre reputación corporativa y creación de valor económico patrimonial”. Perspectiva Empresarial 2 (2015): 85-95. http://dx.doi. org/10.16967\%2Frpe.v2n1a6

\section{MLA:}

Daza-Izquierdo, Julio. “Análisis de la relación circular entre reputación corporativa y creación de valor económico patrimonial". Perspectiva Empresarial 2.1 (2015): 8595. Digital. http://dx.doi. org/10.16967\%2Frpe.v2n1a6 management as they legitimize the integration of corporate social responsibility practices in big Spanish companies sought-after in the stock market.

KEYWORDS shareholders, value creation, panel data, interest groups, Tobin's Q Ratio, corporate reputation.

\section{Análise da relação circular entre reputação corporativa e criação de valor econômico patrimonial}

RESUMO Determinar se as companhias devem incorporar aspectos sociais em suas estratégias de gestão conducentes para satisfazer as expectativas de seus distintos stakeholders apresenta posturas teóricas e evidência empírica prévia tanto a favor quanto contra. 0 objetivo do presente estudo consiste em analisar a relação existente entre a reputação corporativa e a criação de valor econômico patrimonial nas empresas cotadas no mercado da bolsa de valores espanhola durante o período 2000-2012 aplicando uma metodologia de dados de painel. Os resultados obtidos indicam-nos que a reputação influi positivamente na criação de valor econômico patrimonial, e que, ao mesmo tempo, um maior valor empresarial repercute em uma maior reputação corporativa, dando mostras de uma relação bidirecional que retroalimenta as duas variáveis objeto de estudo. Estes resultados têm importantes implicações para a gestão, já que legitimam a integração de práticas de responsabilidade social corporativa nas grandes empresas espanholas cotadas na bolsa.

PALAVRAS CHAVE acionistas, criação de valor, dados de painel, grupos de interesse, proporção q de Tobin, reputação corporativa. 


\section{Introducción}

La reputación corporativa se suele identificar con la imagen pública o de marca de una compañía. Sin embargo, este es un concepto mucho más amplio que implica no solo la imagen externa sino también la imagen interna que tienen los distintos grupos de interés sobre la compañía. Concretamente, la reputación es el resultado de un amplio proceso que se inicia con el comportamiento de la compañía con sus distintos participantes. Posteriormente intervienen intermediarios especializados, que analizan la información dispersa y le dan difusión. Por último, son los propios agentes económicos los que evalúan a la compañía y generan percepciones a partir de su relación con ella y de la información que reciben de los intermediarios especializados.

En este sentido, se puede definir la reputación corporativa como la percepción generalizada que se tiene sobre la capacidad de una compañía de satisfacer las expectativas de sus distintos stakeholders, tanto en términos de comportamiento como de transparencia informativa. Se trata, por consiguiente, de un activo intangible de gran relevancia que, en los últimos años, ha suscitado el interés no solo de los académicos sino también de los profesionales, sean ellos gestores, analistas o inversores. Además, la reputación corporativa es fuente de ventajas competitivas porque cumple las cualidades de ser un recurso estratégico y una capacidad dinámica que proporciona valor al utilizarse de forma estratégica (Martínez \& Olmedo, 2010).

Desde el punto de vista académico, existe un encendido debate tanto teórico como empírico acerca de la necesidad de incorporar la relación con los stakeholders en las estrategias de gestión. Por otro lado, desde un punto de vista profesional, los directivos de las empresas están interesados en conocer las implicaciones de incorporar los principios de responsabilidad social en sus compañías, con el fin de mejorar las relaciones estratégicas con sus grupos de interés. También los inversores institucionales y gestores de carteras están cada vez más interesados por el impacto bursátil de estas medidas, y por saber si este tipo de gestión puede aumentar el valor de mercado de la compañía.

Sin embargo, la evidencia empírica previa que analiza la naturaleza de la relación entre reputación corporativa y la creación de valor económico patrimonial no presenta resultados concluyentes.
Esto se debe a una serie de limitaciones, como son la falta de fundamentos teóricos, el uso de variables inapropiadas para medir reputación o valor, la especificación de modelos simplistas que proporcionan estimaciones no robustas que pueden dar lugar a falsos resultados, y el empleo de bases de datos que muestran una mínima variabilidad en cuanto a las características subyacentes de las compañías. En este contexto, el objetivo del presente estudio consiste en aportar evidencia empírica adicional que supere las limitaciones indicadas anteriormente, y que proporcione resultados robustos con respecto a la naturaleza de la relación entre reputación corporativa y creación de valor económico patrimonial en el mercado bursátil español durante los años 2000 a 2012.

Una de las contribuciones del presente trabajo consiste en el análisis de un amplio periodo temporal que supera los diez años de estudio, en los que se incluyen unos primeros años de expansión, así como años caracterizados por una fuerte crisis financiera internacional. Con el objeto de controlar dichos efectos macroeconómicos, al igual que los efectos específicos de cada empresa, se optó por realizar un análisis con datos de panel aplicando diversos métodos como los tradicionalmente empleados: mínimos cuadrados ordinarios y efectos fijos o aleatorios, así como el método generalizado de los momentos. Todo ello permite proporcionar resultados concluyentes acerca de la relación entre las dos variables objeto de estudio.

Concretamente, los resultados obtenidos indican que la reputación influye de manera positiva en la creación de valor económico patrimonial, y que, simultáneamente, un mayor valor empresarial repercute en una mayor reputación corporativa, dando muestras de una relación bidireccional que retroalimenta las dos variables objeto de estudio. Estos resultados tienen importantes implicaciones para la gestión, dado que legitiman la integración de prácticas de responsabilidad social en las grandes empresas españolas, como se comentará a lo largo del texto.

El resto del trabajo se estructura del siguiente modo. En el segundo apartado se describen las posturas teóricas a favor y en contra de la incorporación de aspectos sociales en las estrategias de gestión. En el tercero, se describen las hipótesis objeto de estudio acerca de la naturaleza de la relación entre estrategia social y valor económico patrimonial. El conjunto de empresas españolas con mayor reputación durante el periodo 20002012 se presenta en el apartado siguiente. En el 
quinto, se presentan los resultados obtenidos del análisis de la relación entre reputación y creación de valor, y en el apartado sexto se describen las conclusiones e implicaciones para la gestión, que derivan del conjunto del trabajo.

\section{La teoría de los stakeholders y la creación del valor inteligente}

La cuestión de si las compañías deben incorporar aspectos sociales en sus estrategias de gestión conducentes a satisfacer las expectativas de sus distintos stakeholders presenta posturas teóricas tanto a favor como en contra. Por un lado, se encuentra la postura neoclásica, defendida por Friedman (1970), que sostiene que la responsabilidad de la empresa es usar sus recursos en actividades encaminadas a maximizar sus beneficios, actuando conforme a las reglas básicas de la sociedad incorporadas en la ley, y a las costumbres éticas. Por tanto, la función social de la empresa se debe limitar a que el valor de los resultados obtenidos no sea inferior al de los recursos empleados. Esto se consigue cuando las empresas maximizan sus beneficios o cuando maximizan el valor del capital de los propietarios o accionistas. De ahí la conclusión de que las empresas deben maximizar dicho valor. Cuando esto se logra, según Friedman (1970), se estará optimizando la aportación de la empresa a la sociedad. Lo anterior implica que cualquier otra actividad que le impida a la empresa maximizar el valor económico patrimonial se considerará inaceptable, ya que se estará incurriendo en una mala asignación de recursos.

La postura neoclásica sostiene que la gestión de la empresa debe girar solo y exclusivamente en el interés de sus propietarios o accionistas. En contraposición a dicha postura se encuentra la denominada teoría de los stakeholders, desarrollada por Freeman (1984), que considera que la empresa no es cosa de uno (propietario o accionista) sino que debe ser entendida desde la pluralidad de agentes que intervienen en ella y, por tanto, la hacen posible. En este sentido, el objetivo de la empresa no debe ser la maximización del valor de mercado sino que la empresa debe crear valor para todos los stakeholders, incluyendo empleados, consumidores, comunidades locales, recursos naturales o medioambientales, etc.

Siguiendo esta teoría, algunos autores como Post, Preston \& Sachs (2002) sostienen que las empresas deben aplicar aquellos aspectos sociales que sean necesarios, independientemente de los costes en los que se incurran, así como de los ingresos que produzcan. En cambio otros autores, como Jensen (2002), apoyan que el objetivo de la empresa tiene que seguir siendo la maximización del valor económico patrimonial, siendo posible al mismo tiempo implementar medidas de carácter social en la gestión, que deben ser planteadas en términos de creación de valor. Conviene, por ende, saber responder a la pregunta de si estas medidas son rentables para la empresa, en el sentido de que permitan maximizar el valor para los accionistas, lo que Jensen (2002) denomina "la creación de valor inteligente".

En este contexto, el objetivo del presente estudio consiste en analizar si las políticas de estrategia social adoptadas en las grandes empresas españolas, medidas a través de su reputación corporativa, pueden crear, destruir o no afectar de manera considerable el valor de mercado de la empresa -y, por tanto, de sus accionistas-, contribuyendo o no a la generación del denominado "valor inteligente" en la empresa.

\section{Planteamiento teórico de la relación entre reputación y creación de valor económico patrimonial}

Antes de realizar el análisis empírico, es necesario exponer las diferentes alternativas que podrían existir en cuanto a la relación entre reputación y creación de valor, así como las hipótesis sobre las que se sustentan dichos posibles vínculos. Para ello se recurrió al marco conceptual desarrollado por Preston \& O’Bannon (1997) -y posteriormente ampliado por Gómez (2008)para resumir los fundamentos teóricos de la relación entre performance social y financiera; estos se recogen en la tabla 1, donde se exponen las distintas alternativas de estudio, así como los posibles resultados que podrían proporcionar, y el nombre de las hipótesis a las que están asociados dichos resultados.

Siguiendo a Preston \& O’Bannon (1997), se podrían distinguir tres posibles alternativas para el estudio de la relación entre reputación (entendida como resultado de la actuación social de la empresa) y creación de valor. En primer lugar, el planteamiento más extendido que analiza tan solo 
TABLA 1. Hipótesis alternativas de la relación entre performance social y financiera

\begin{tabular}{llll}
\hline & SIGNO DE LA RELACIÓN & Negativa \\
\cline { 2 - 2 } & Positiva & Neutral & Hipótesis del trade-off \\
\hline Social $\rightarrow$ Financiera & Hipótesis del impacto social & Hipótesis de las variables \\
\cline { 1 - 2 } Social $\leftarrow$ Financiera & $\begin{array}{l}\text { Hipótesis de la disponibilidad de } \\
\text { fondos }\end{array}$ & $\begin{array}{l}\text { Hipótesis del oportunismo } \\
\text { de los directivos }\end{array}$ \\
\cline { 1 - 2 } Social $\leftrightarrow$ Financiera & Sinergia positiva & Sinergia negativa \\
\hline
\end{tabular}

Fuente: Preston \& O’Bannon (1997) y Gómez (2008)

el posible impacto de la reputación corporativa sobre la creación de valor económico patrimonial de la empresa. Una segunda alternativa de estudio sería el análisis de la influencia de la creación de valor sobre las políticas de responsabilidad social encaminadas a mejorar las relaciones con los distintos participantes en la empresa. Una última alternativa, menos extendida, consistiría en el análisis de las sinergias existentes entre ambas variables.

Concretamente, cuando la creación de valor es la variable dependiente, y el objetivo del estudio es la influencia de la reputación como resultado de las políticas de responsabilidad social, existen dos hipótesis alternativas para explicar los posibles resultados. Por un lado, un resultado positivo vendría explicado por la hipótesis de impacto social, que sostiene que cuando la empresa sea capaz de gestionar de forma eficaz las relaciones con sus grupos de interés, obtendrá una ventaja competitiva que le permitirá generar valor para la empresa a largo plazo (Freeman, 1984; Agnihotri, 2014). Por otro lado, un resultado negativo vendría explicado por la hipótesis del trade-off, la cual sostiene que los altos costes de las acciones de responsabilidad social suponen una disminución notable de los beneficios de las empresas, y las sitúa en una situación de desventaja frente a otras, que les impedirá generar valor económico patrimonial (Friedman, 1970).

Por el contrario, en el caso de ser la reputación de la empresa la variable dependiente, siendo por tanto el objetivo del análisis la influencia de la creación de valor en ella, son otras dos las hipótesis alternativas que se pueden considerar para explicar los posibles resultados por encontrar. La relación positiva vendría explicada por la denominada hipótesis de disponibilidad de fondos (Waddock \& Graves, 1997), según la cual, cuanto mayor es la creación de valor en la empresa, mayores son las posibilidades de satisfacer de forma adecuada los intereses de todos sus participantes o grupos de interés, lo que favorecerá la consolidación de la reputación de la empresa. Por su parte, un resultado negativo tendría su fundamento teórico en la hipótesis del oportunismo de los directivos, que plantea que los objetivos que persiguen los directivos pueden ser distintos a los de los propietarios y a los de los demás grupos de interés (Williamson, 1985), ya que mientras que los objetivos de los directivos estarían orientados al corto plazo y a los beneficios inmediatos, los objetivos de los propietarios o de los accionistas estarían más ligados al largo plazo.

Por último, como señalan Preston \& O’Bannon (1997), si ambos tipos de análisis diesen un resultado de signo positivo, se estaría hablando de la existencia de sinergias positivas entre ambas variables. En cambio, si los resultados hubiesen sido del signo contrario, se estaría aceptando la existencia de sinergias de carácter negativo. Sin embargo, por tradición, la evidencia empírica previa ha encontrado una relación de carácter positivo entre ambas variables (Brown, 1997; McMillan \& Joshi, 1997; Srivastava et al., 1997; Brown, 1998; Vergin \& Qoronfleh, 1998; Black et al., 2000; Jiao, 2010) cuando las muestras analizadas correspondían a empresas norteamericanas. Mientras que el análisis efectuado en otros países como Dinamarca (Rose \& Thomsen, 2004), Alemania (Eberl \& Schwaiger, 2005) o Australia (Inglis et al., 2006) indica la existencia de una relación neutra. En este sentido, cabe destacar el trabajo de Gómez (2008), que añade la hipótesis de las variables moderadoras a las ya recogidas por Preston \& O’Bannon (1997) para explicar la neutralidad o la falta de significatividad encontrada en la relación entre ambas variables sobre la base de otras variables explicativas que podrían estar moderando dicha relación. Por ello, Gómez (2008) considera que el análisis multivariante es el marco más adecuado para discernir entre las hipótesis anteriormente mencionadas. En el contexto de la literatura que se acaba de mencionar, el objetivo del presente estudio es analizar qué tipo de relación existe entre reputación y creación de valor económico 
patrimonial, aplicado también para el caso de las compañías cotizadas en la bolsa española.

\section{Las empresas españolas con mayor reputación}

Para contrastar las hipótesis propuestas en el apartado anterior es preciso conocer cuáles son las empresas españolas cotizadas en bolsa con mayor reputación corporativa. La información sobre reputación corporativa ha sido obtenida del ranking merco. Este índice proporciona una puntuación de las 100 compañías en España con la mayor reputación corporativa desde el año 2001 hasta la actualidad ${ }^{1}$.

Este ranking es similar al proporcionado por Fortune, y es ampliamente seguido por la evidencia empírica previa en nuestro país (Fernández \& Luna, 2007; Delgado et al., 2010 y 2013; Baraibar y Luna, 2012). Las variables consideradas por merco para la elaboración de su ranking anual son seis: resultados económico-financieros, calidad de la oferta comercial, reputación interna, ética y responsabilidad corporativa, dimensión internacional de la empresa e innovación. Estas, a su vez, se desagregan en tres subapartados, como se indica en la tabla 2.

La metodología empleada para la elaboración de dicho ranking consiste, en primer lugar, en la realización de una encuesta a los miembros de los comités de dirección de todas las empresas que facturan anualmente en España más de 50 millones de euros. En segundo lugar, se hace una evaluación de expertos: a) miembros del Instituto de Analistas Financieros, quienes evalúan los resultados económico-financieros y la calidad de la información económica; b) las asociaciones de consumidores ${ }^{2}$, que evalúan la calidad de producto y servicio y el respeto a los derechos del consumidor; c) personas vinculadas

1 Aunque el primer informe se hizo público en el primer trimestre de 2001, este hacía referencia al ejercicio económico 2000, año en el que se inició el presente estudio.

2 Confederación Española de Organizaciones de Amas de Casa, Consumidores y Usuarios (Ceaccu), la Confederación Española de Cooperativas Consumidores y Usuarios (Hispacoop), la Asociación de Usuarios de la Comunicación (AUC), la Confederación de Consumidores y Usuarios (CECU).
TABLA 2. Variables consideradas para la elaboración del ranking merco

\begin{tabular}{ll}
\hline $\begin{array}{l}\text { Resultados } \\
\text { económico- } \\
\text { financieros }\end{array}$ & $\begin{array}{l}\text { Beneficios contables } \\
\text { Rentabilidad } \\
\text { Calidad de la información económica }\end{array}$ \\
\hline $\begin{array}{l}\text { Calidad de la } \\
\text { oferta comercial }\end{array}$ & $\begin{array}{l}\text { Valores de producto } \\
\text { Valores de marca } \\
\text { Servicio y atención al cliente }\end{array}$ \\
\hline Reputación & $\begin{array}{l}\text { Calidad laboral } \\
\text { Valores éticos y profesionales } \\
\text { Identificación con el proyecto } \\
\text { empresarial }\end{array}$ \\
\hline $\begin{array}{l}\text { Ética y } \\
\text { responsabilidad } \\
\text { corporativa }\end{array}$ & $\begin{array}{l}\text { Comportamiento corporativo ético } \\
\text { Responsabilidad social y } \\
\text { medioambiental }\end{array}$ \\
\hline $\begin{array}{l}\text { Dimensión } \\
\text { internacional de } \\
\text { la empresa }\end{array}$ & $\begin{array}{l}\text { Número de países en los que opera } \\
\text { Cifra de negocio en el extranjero } \\
\text { Alianzas estratégicas internacionales }\end{array}$ \\
\hline Innovación & $\begin{array}{l}\text { Inversiones en I+D } \\
\text { Nuevos productos y servicios } \\
\text { Nuevos canales }\end{array}$ \\
\hline
\end{tabular}

Fuente: Monitor español de reputación corporativa

a entidades no lucrativas ${ }^{3}$, que evalúan el compromiso con la comunidad y la responsabilidad social y medioambiental; d) los sindicatos, que evalúan la reputación interna y la calidad laboral; e) los líderes de opinión, que evalúan el ranking provisional de líderes. Posteriormente, se incorporan los resultados de ranking-merco con el análisis de la perspectiva del consumidor. En cuarto lugar, se añaden los resultados de merco-personas con el análisis de la perspectiva de los trabajadores. Por último, los técnicos del instituto responsable, Análisis e Investigación, verifican mediante un "cuestionario de méritos" la reputación de cada una de las empresas que figuran en el ranking provisional de reputación corporativa ${ }^{4}$.

Esta laboriosa metodología empleada por el monitor español permite disponer de una medida agregada de la percepción de los distintos

3 Fundación Ecología y Desarrollo, Ayuda en Acción, Asociación Española de Fundaciones, Cermi, Confederación Iberoamericana de Fundaciones, Cruz Roja, Fundación + familia, Profesionales por la Ética, Save The Children, Sociedad Española de Ornitología, Solidarios para el Desarrollo, Transparency International (dos entidades no lucrativas prefieren mantener su anonimato).

4 Los resultados de dichos informes anuales pueden ser consultados en www.merco.info/es 
TABLA 3. Empresas con mayor reputación corporativa

\begin{tabular}{|c|c|c|}
\hline INDUSTRIA & SECTOR & NOMBRE \\
\hline \multirow{2}{*}{ Bienes de consumo } & Alimentación & Campofrío, Ebro Puleva \\
\hline & Tabaco & Altadis \\
\hline \multirow{3}{*}{ Financiero } & Bancos & $\begin{array}{l}\text { Banco Popular, Banco Sabadell, Banco Santander, Banesto, } \\
\text { Bankia, Bankinter, BBVA, Caixabank }\end{array}$ \\
\hline & Seguros & Catalana Occidente, Mapfre \\
\hline & Inversiones y servicios inmobiliarios & Inmobiliaria Colonial, Fadesa, Urbis, Metrovacesa \\
\hline \multirow{3}{*}{ Industria } & Construcción & $\begin{array}{l}\text { Abengoa, Acciona, ACS, Dragados, Ferrovial, FCC, OHL, } \\
\text { Portland, Sacyr Vallehermoso }\end{array}$ \\
\hline & Transporte & Abertis, EADS \\
\hline & Servicios de seguridad & Prosegur \\
\hline \multirow{2}{*}{ Petróleo y gas } & Energías alternativas & Gamesa \\
\hline & Productores de petróleo y gas & Petróleos (Cepsa), Repsol YPF \\
\hline Salud & Farmacia y biotecnología & Grifols, Zeltia \\
\hline \multirow{3}{*}{ Servicios de consumo } & Confección & Adolfo Domínguez, Carrefour, Cortefiel, Inditex \\
\hline & Medios de comunicación & Antena 3, Prisa, Vocento \\
\hline & Viajes y ocio & Iberia, NH Hoteles, Sol Meliá, Vueling \\
\hline Tecnología & Software y servicios informáticos & Indra \\
\hline \multirow{2}{*}{ Telecomunicaciones } & Telecomunicaciones de línea fija & Telefónica \\
\hline & Telecomunicaciones móviles & Telefónica Móviles \\
\hline \multirow{2}{*}{ Utilities } & Gas y agua & Enagas, Gas Natural \\
\hline & Electricidad & Endesa, Iberdrola, Unión Fenosa, Red Eléctrica Corporación \\
\hline
\end{tabular}

Fuente: Base de datos DataStream

stakeholders sobre las compañías analizadas. No obstante, es preciso señalar que no todas las compañías con mayor reputación cotizan en la bolsa española. Uno de los motivos es que se trata de multinacionales que cotizan en sus mercados de origen y no en el mercado español (ejemplos de ello son: Coca-cola, IBM, Siemens, etc.). Otro motivo se encuentra en la decisión de no cotizar en bolsa de grandes compañías españolas como El Corte Inglés o Mercadona, habituales en el ranking. Salvo estas excepciones, en la tabla 3 se presentan las empresas cotizadas españolas incluidas por el ranking merco entre los años de estudio, clasificadas en función del sector industrial al que pertenecen. Como se puede observar, los sectores más ampliamente representados son el financiero, el industrial, el de servicios de consumo y el de utilities. A dichos sectores también pertenecen las compañías incluidas en el ranking en todos los años objeto de estudio; estas son: ACS, Banco Popular, Banco Santander, Bankinter, BBVA, Endesa, Ferrovial, Gamesa, Gas Natural, Iberdrola, Mapfre, Repsol YPF, Sol Meliá y Telefónica. Tal información muestra la persistencia de esta cualidad en las grandes empresas españolas.

\section{Naturaleza de la relación entre reputación y creación de valor económico patrimonial}

Una vez conocidas las empresas españolas cotizadas con mayor reputación, el objetivo es evaluar la relación con la creación de valor económico patrimonial. Para ello se empleó el ratio q de Tobin, el cual relaciona la valoración que hace el mercado acerca de la capacidad de generar rentabilidad para el accionista (medida esta por la capitalización bursátil o valor de mercado de la empresa) sobre el coste de reposición de los activos (esto es, el coste que supondría adquirir la capacidad productiva de la empresa) aproximado por el valor de mercado de los fondos propios y la deuda entre el valor contable de los activos. De este modo, si el ratio q es superior a la unidad, la empresa estará creando valor, puesto que el mercado está haciendo una valoración de la empresa superior al coste de reposición de sus activos productivos. En caso contrario se destruye valor. Como señala Jiao (2010), este ratio es el más adecuado para este tipo de estudio ya que, al igual que la reputación corporativa, es una variable basada no solo en datos históricos sino también 
TABLA 4. Variables consideradas en el estudio

\begin{tabular}{ll}
\hline NOMBRE & CÁLCULO \\
\hline Variables por analizar & $\begin{array}{l}\text { Logaritmo neperiano de la puntuación otorgada por el monitor español de reputación } \\
\text { Corporativa cada año. }\end{array}$ \\
\hline $\begin{array}{l}\text { Creación de valor económico } \\
\text { patrimonial }\end{array}$ & $\begin{array}{l}\text { Valor de mercado de los fondos propios y la deuda entre el valor contable de los } \\
\text { activos (aproximación al ratio q de Tobin). }\end{array}$ \\
\hline Variables de control & Logaritmo neperiano del valor del activo total de la empresa. \\
\hline Tamaño & Deuda a largo plazo sobre el activo total. \\
\hline Endeudamiento & $\begin{array}{l}\text { Rentabilidad sobre activos, calculada como el cociente entre el beneficio económico } \\
\text { yel activo total de la empresa. }\end{array}$ \\
\hline
\end{tabular}

Fuente: elaboración propia

en expectativas. Esta es su principal ventaja con respecto a otras alternativas, como podrían ser los ratios de rentabilidad basados exclusivamente en la información suministrada por los estados financieros de la empresa, que están siendo paulatinamente desplazados en los estudios empíricos. No obstante, teniendo en cuenta la sugerencia de Gómez (2008) descrita en el tercer apartado, también ha sido considerada en el estudio una serie de variables moderadoras o de control, que se describe en la tabla 4. Siguiendo la evidencia empírica más reciente (Jiao, 2010), se empleó una variable de tamaño, para controlar por posibles efectos de escala; una variable de endeudamiento, para controlar por el impacto de la estructura de capital, y una variable de rentabilidad, para controlar por el momento económico que atraviesa la empresa ${ }^{5}$.

Del mismo modo, se examinó la relación de causalidad entre ambas variables mediante el contraste de causalidad de Granger. El valor del estadístico chi-cuadrado de Pearson obtenido $(2,729)$ indica la existencia de una relación de causalidad de la reputación sobre la creación de valor de signo positivo y significativo a los niveles convencionales de confianza.

Con el objeto de presentar resultados concluyentes, las estimaciones se han realizado mediante la técnica de datos de panel, como muestran las siguientes fórmulas:

Creación valor $_{i t}=\alpha_{0}+\alpha_{1}$ Reputación corporativa $_{i t}+\sum_{\mathrm{j}=2}^{4} \alpha_{j} \mathrm{CV}_{\mathrm{jit}}+\mu_{\mathrm{t}}+\eta_{i}+\varepsilon_{i t}$
Reputación corporativa $_{i t}=\alpha_{0}+\alpha_{1}$ Creacionvalor $_{i t}+\sum_{\mathrm{j}=2}^{4} \alpha_{j} \mathrm{CV}_{\mathrm{jit}}+\mu_{\mathrm{t}}+\eta_{i}+\varepsilon_{i t}$

A diferencia del análisis de sección cruzada, este tipo de análisis permite modelizar la heterogeneidad inobservable que existe entre distintas

5 Toda esta información se obtuvo de la base de datos DataStream. empresas. Esto se consigue mediante la descomposición del término de error en tres componentes: un primer componente representativo de cada empresa que recoge aquellos efectos no observables que le afectan solo a ella (heterogeneidad inobservable); un segundo componente representativo de los shocks acaecidos en cada año de estudio y que afectan a todas las empresas por igual (efectos macroeconómicos), y un tercer componente que es una variable aleatoria. Además, permite ampliar la muestra, lo que significó un aumento sustancial de las observaciones, de los grados de libertad del modelo y, por tanto, de la consistencia de los resultados. Adicionalmente, y con el objeto de presentar resultados robustos, el análisis con datos de panel se llevó a cabo empleando tanto el estimador de mínimos cuadrados ordinarios y el de efectos fijos o aleatorios, como el estimador de sistema del método generalizado de los momentos (System GMM) que emplea variables instrumentales para estimar el modelo de forma consistente (Arellano \& Bond, 1991) evitando, con ello, el problema de endogeneidad en las variables ${ }^{6}$.

Los resultados obtenidos se presentan en las tablas 5 y 6 . Concretamente, en la tabla 5 , aparecen los resultados de estimar la creación de valor en función de la reputación corporativa y las variables de control anteriormente señaladas, mientras que en la tabla 6 se exponen los resultados de cuando la variable dependiente es la reputación corporativa. Es preciso indicar que en ambos casos se incluyó la variable dependiente retardada un periodo debido a su carácter persistente ${ }^{7}$.

6 Correlación con el término de error.

7 Esta es otra de las medidas adoptadas en el presente estudio para superar las limitaciones metodológicas observadas en estudios precedentes. 
TABLA 5. Variables explicativas de la creación de valor económico patrimonial

\begin{tabular}{|c|c|c|c|}
\hline & $\begin{array}{c}\text { MÍNIMOS CUADRADOS } \\
\text { ORDINARIOS }\end{array}$ & $\begin{array}{l}\text { EFECTOS FIJOS O } \\
\text { ALEATORIOS }\end{array}$ & GMM POR SISTEMAS \\
\hline Constante & $-1,100$ & $-1,100^{* * *}$ & $-0,840$ \\
\hline Creación de valor (-1) & $0,466^{* * *}$ & $0,466^{* * *}$ & $0,479^{* \star *}$ \\
\hline Reputación corporativa & $0,451^{* * *}$ & $0,451^{* \star *}$ & $0,417^{*}$ \\
\hline Tamaño & $-0,150^{\star * *}$ & $-0,150^{\star \star \star}$ & $-0,145^{* *}$ \\
\hline Endeudamiento & 0,142 & 0,142 & 0,226 \\
\hline Rentabilidad & 0,865 & $0,865^{*}$ & $-0,072$ \\
\hline $\mathrm{R}^{2}$ & 0,7063 & 0,7063 & \\
\hline Test de Wald & $139,34^{* * *}$ & $362,60^{* * *}$ & \\
\hline Test de Hausman & & 0,00 & \\
\hline $\mathrm{m}_{1}$ & & & 1,62 \\
\hline $\mathrm{m}_{2}$ & & & 1,20 \\
\hline $\mathrm{z}_{1}$ & & & $71,93^{* \star *}$ \\
\hline $\mathrm{z}_{2}$ & & & $5,01^{* * *}$ \\
\hline Test de Sargan & & & 772,16 \\
\hline
\end{tabular}

Nota: Esta tabla presenta los resultados empleando el método de mínimos cuadrados ordinarios (MCO), efectos fijos o aleatorios, así como el método generalizado de los momentos (GMM) por sistemas. Los resultados que se muestran de cada una de las variables corresponden al valor de sus coeficientes acompañado por un indicativo si son significativos estadísticamente.

Para los dos primeros contrastes se muestran además los resultados del coeficiente de determinación $\mathrm{R}^{2}$ para medir la bondad del modelo, el test de Wald para testar si los parámetros de las variables explicativas son igual a cero y el test de Hausman para decidir entre la elección de efectos fijos o efectos aleatorios. Por último, para la estimación por GMM se proporcionan los contrastes $m_{1}$ y $m_{2}$ de ausencia de correlación serial de primer y segundo orden, respectivamente, de los residuos del modelo en primeras diferencias; el test $z_{1}$ de significatividad conjunta de los coeficientes de las variables explicativas del modelo; el test $z_{2}$ de significatividad conjunta de las dummies de año incluidas en el modelo, y el test de Sargan por el que se contrasta la sobreidentificación de los instrumentos utilizados.

***, ${ }^{* \star} y$ * representan significatividad a un nivel del $1 \%, 5 \%$ y $10 \%$, respectivamente.

Fuente: elaboración propia

TABLA 6. Variables explicativas de la reputación corporativa

\begin{tabular}{|c|c|c|c|}
\hline & $\begin{array}{c}\text { MÍNIMOS CUADRADOS } \\
\text { ORDINARIOS }\end{array}$ & $\begin{array}{l}\text { EFECTOS FIJOS } \\
\text { O ALEATORIOS }\end{array}$ & GMM POR SISTEMAS \\
\hline Constante & $1,432^{* * *}$ & $4,178^{* * *}$ & $1,495^{*}$ \\
\hline Reputación corporativa (-1) & $0,733^{\star \star *}$ & $0,466^{\star * \star}$ & $0,732^{* * *}$ \\
\hline Creación de valor & $0,058^{\star \star \star}$ & $0,058^{* *}$ & $0,061^{* \star *}$ \\
\hline Tamaño & $0,043^{* * *}$ & 0,001 & $0,048^{* * *}$ \\
\hline Endeudamiento & $-0,102$ & 0,083 & $-0,118$ \\
\hline Rentabilidad & 0,126 & $-0,954^{* *}$ & $-0,076$ \\
\hline $\mathrm{R}^{2}$ & 0,8496 & 0,8924 & \\
\hline Test de Wald & $139,34^{\star \star \star}$ & $362,60^{* * *}$ & \\
\hline Test de Hausman & & $97,05^{* * *}$ & \\
\hline$m_{1}$ & & & $-0,84$ \\
\hline $\mathrm{m}_{2}$ & & & 0,74 \\
\hline$z_{1}$ & & & $6,03^{\star \star \star}$ \\
\hline $\mathrm{z}_{2}$ & & & $8,19^{* * \star}$ \\
\hline Test de Sargan & & & $277,25^{\star \star \star}$ \\
\hline
\end{tabular}

Nota: Esta tabla presenta los resultados empleando el método de mínimos cuadrados ordinarios (MCO), efectos fijos o aleatorios, así como el método generalizado de los momentos (GMM) por sistemas. Los resultados que se muestran de cada una de las variables corresponden al valor de sus coeficientes acompañado por un indicativo si son significativos estadísticamente.

Para los dos primeros contrastes se muestran además los resultados del coeficiente de determinación $\mathrm{R}^{2}$ para medir la bondad del modelo, el test de Wald para testar $\mathrm{s}$ los parámetros de las variables explicativas son igual a cero y el test de Hausman para decidir entre la elección de efectos fijos o efectos aleatorios. Por último, para la estimación por GMM se proporcionan los contrastes $\mathrm{m}_{1}$ y $\mathrm{m}_{2}$ de ausencia de correlación serial de primer y segundo orden, respectivamente, de los residuos del modelo en primeras diferencias; el test $z_{1}$ de significatividad conjunta de los coeficientes de las variables explicativas del modelo; el test $z_{2}$ de significatividad conjunta de las dummies de año incluidas en el modelo, y el test de Sargan por el que se contrasta la sobreidentificación de los instrumentos utilizados.

$\star * \star, * * y *$ representan significatividad a un nivel del $1 \%, 5 \%$ y $10 \%$, respectivamente.

Fuente: elaboración propia 
Como se puede observar en la tabla 5, la reputación corporativa influye positivamente en la creación de valor económico patrimonial, dando soporte a la hipótesis de impacto social defendida por Freeman (1984). Por otro lado, del contenido de la tabla 6 se deduce que un mayor valor empresarial también influye positivamente, aunque en menor medida, en el incremento de la reputación corporativa, y, por tanto, en el grado de satisfacción de los distintos stakeholders, lo cual corrobora de esta manera la hipótesis de disponibilidad de fondos expuesta por Waddock \& Graves (1997). Es importante también hacer énfasis en que ambos conjuntos de resultados son independientes del método empleado para realizar las estimaciones, así como de la consideración de variables moderadoras como son el tamaño, el nivel de endeudamiento y de resultados de las compañías.

Siguiendo la evidencia empírica previa, se contrastó la correcta especificación de las estimaciones utilizando el coeficiente de determinación, así como el contraste de significatividad conjunta de las variables explicativas cuando las estimaciones han sido realizadas por mínimos cuadrados o aplicando efectos fijos o aleatorios. En cambio, cuando las estimaciones se realizaron empleando GMM por sistemas, la correcta especificación se contrastó empleando en primer lugar el estadístico de Hansen de restricciones de sobre-identificación, con el fin de contrastar la ausencia de correlación entre los instrumentos y el término de error, y se confirmó que los instrumentos empleados son válidos. En segundo lugar, se utilizó el estadístico desarrollado por Arellano \& Bond (1991) para contrastar la ausencia de correlación serial de segundo orden en los residuos en primeras diferencias, y se observó que no existen problemas de correlación serial de segundo orden en nuestros modelos. Por último, se obtuvieron unos resultados adecuados en los contrastes de significatividad conjunta de los coeficientes de las variables explicativas y de las variables dicotómicas temporales.

En definitiva, cabe señalar que para las compañías cotizadas españolas con mayor reputación se observa durante el periodo 2000-2012 la existencia de sinergias positivas entre su grado de atención a los distintos grupos de interés y la creación de valor para los propietarios o accionistas. Estos resultados son contundentes y ponen de manifiesto que la atención a los distintos stakeholders no está reñida con la creación de valor para la empresa y sus accionistas, sino todo lo contrario: que estos dos factores se retroalimentan generando con ello un efecto circular creciente.

\section{Conclusiones e implicaciones para la gestión}

La evidencia obtenida en el presente estudio es de vital importancia para la gestión empresarial, ya que elimina dudas sobre el debate existente entre la relevancia de incluir o no medidas sociales en las estrategias de gestión, y supone un argumento de legitimación interno para las empresas en lo que se refiere a la integración de estas prácticas de responsabilidad social en su gestión estratégica. Concretamente, nuestro estudio ofrece una imagen clara de la influencia de la satisfacción de los stakeholders en la creación de valor para la empresa, y por tanto para sus accionistas, mientras que una mayor creación de valor económico patrimonial repercute también positivamente, aunque en menor medida, en los niveles de reputación empresarial, existiendo por tanto sinergias positivas entre ambas magnitudes. Estos resultados, por consiguiente, ponen de manifiesto que el éxito en la gestión se conseguirá sabiendo equilibrar dos conceptos básicos: la relación con los grupos de interés y la creación de riqueza.

También es preciso indicar las implicaciones de este estudio para los inversores. Concretamente, cuando los agentes económicos toman sus decisiones de inversión en bolsa tratan de colocar el capital en las empresas con mejores perspectivas de futuro. En este sentido, la reputación corporativa puede ser entendida como un indicador de la capacidad que va a tener la empresa de acceder a recursos de calidad superiores en condiciones favorables y, por tanto, como un anticipo de la generación de valor para la empresa y sus accionistas. Estos resultados además motivarán a otros inversores a elegir las compañías más reputadas para sus futuras inversiones, ya que se demuestra en este estudio que una mayor atención a los distintos stakeholders supone mayor valor y viceversa.

Una vez obtenidos estos resultados, investigaciones futuras deberían ir encaminadas al análisis de la influencia desagregada de la satisfacción de cada grupo de interés en la creación de valor de la empresa, así como de la influencia de la creación de valor en cada uno de estos grupos de interés. Este tipo de análisis nos permitirá profundizar en el conocimiento del proceso de generación de 
valor para los accionistas, y encontrar argumentos específicos para los gestores que contribuyan a satisfacer al resto de agentes integrantes en la empresa.

\section{Referencias}

Agnihotri, A. (2014). Mass-media-based Corporate Reputation and firms's market valuation. Evidence from emerging markets. Corporate Reputation Review, 17, 206-218.

Arellano, M. \& Bond, S. (1991). Some tests of specification for panel data: Monte Carlo evidence and application to employment equations. Review of Economic Studies, 58, 277-297.

Baraibar, E. \& Luna, L. (2012). Transparencia social e hipótesis del impacto social. Análisis en el IBEX35. Universia Business Review, 36, 108-123.

Black, E. L., Carnes, T. A. \& Richardson, V. J. (2000). The market valuation of corporate reputation. Corporate Reputation Review, 3(1), 31-42.

Brown, B. (1997). Stock market valuation of reputation for corporate social performance. Corporate Reputation Review, 1(1), 76-80.

Brown, B. (1998). Do stock market investors reward companies with reputations for social performance? Corporate Reputation Review, 1(3), 271-280.

Delgado, J. B., Quevedo, E. de \& Díez, J. M. (2013). The impact of corporate reputation on firm risk: A panel data análisis of Spanish quoted firms. British Journal of Management, 24, 1-20.

Delgado, J. de \& Fuente, J. M. de la (2010). The impact of ownership structure on corporate reputation. Evidence from Spain. Corporate Gobernance: An International Review, 18(6), 540-556.

B., Quevedo, E. Eberl, M. \& Schwaiger, M. (2005). Corporate reputation: Disentangling the effects on financial performance. European Journal of Marketing, 39(7-8), 838-854.

Fernández, J. L. \& Luna, L. (2007). The creation of value through corporate reputation. Journal of Business Ethics, 76, 335-346.

Freeman, R. E. (1984). Strategic management: A stakeholder approach. Boston: Pitman.
Friedman, M. (1970). A Friedman doctrine: The social responsibility of business is to increase its profits. The New York Times Magazine, 13, 32-33.

Gómez, F. (2008). Responsabilidad social corporativa y performance financiero: treinta y cinco años de investigación empírica en busca de un consenso. Principios: Estudios de Economía Política, 11, 5-24.

Inglis, R., Morley, C. \& Sammut, P. (2006). Corporate reputation and organisational performance: an Australian study. Management Auditing Journal, 21(9), 934-947.

Jensen, M. C. (2002). Value maximization, stakeholders theory, and the corporate objective function. Business Ethics Quarterly, 12(2), 235-256.

Jiao, Y. (2010). Stakeholder welfare and firm value. Journal of Banking and Finance, 34, 2549-2561.

Martínez, I. \& Olmedo, I. (2010). Revisión teórica de la reputación en el entorno empresarial. Cuadernos de Economía y Dirección de la Empresa, 44, 59-78.

McMillan, G. S. \& Joshi, M. P. (1997). Sustainable competitive advantage and firm performance: The role of intangible resources. Corporate Reputation Review, 1(1), 81-85.

Post, J., Preston, L. \& Sachs, S. (2002). Redefining the corporation: Stakeholder Management and Organizational Wealth. Stanford, CA: Stanford University Press.

Preston, L. E. \& O'Bannon, D. P. (1997). The Corporate Social-Financial Performance Relationship: A Typology and Analysis. Business and Society, 36, 419-429.

Rose, C. \& Thomsen, S. (2004). The impact of corporate reputation on performance: Some Danish evidence. European Management Journal, 22(2), 201-210.

Srivastava, R. K., McInish, T. H., Wood, R. A. \& Capraro, A. J. (1997). The value of corporate reputation: Evidence from the equity markets. Corporate Reputation Review, 1(1), 62-68.

Vergin, R. C. \& Qoronfleh, M. W. (1998). Corporate reputation and the stock market. Business Horizons, 41(1), 19-26.

Waddock, S. A. \& Graves, S. B. (1997). Corporate Social Performance-Financial Performance Link. Strategic Management Journal, 18(4), 303-319.

Williamson, O. E. (1985). The Economic Institutions of Capitalism. New York: Free Press. 